\title{
Identification of the visceral pain pathway activated by noxious colorectal distension in mice
}

\section{Melinda Kyloh, Sarah Nicholas, Vladimir P. Zagorodnyuk, Simon J. Brookes and Nick J. Spencer*}

Department of Human Physiology, Flinders Medical Science and Technology Cluster, Flinders University, Adelaide, SA, Australia

\section{Edited by:}

Paul P. Bertrand, University of

New South Wales, Australia

Reviewed by:

Christopher Keating, University of Sheffield, UK

Bin Feng, University of Pittsburgh, USA

\section{*Correspondence:}

Nick J. Spencer, Department of Human

Physiology, Flinders University, GPO

Box 2100, Adelaide, SA 5001, Australia.

e-mail: nicholas.spencer@flinders.

edu.au
In patients with irritable bowel syndrome, visceral pain is evoked more readily following distension of the colorectum. However, the identity of extrinsic afferent nerve pathway that detects and transmits visceral pain from the colorectum to the spinal cord is unclear. In this study, we identified which extrinsic nerve pathway(s) underlies nociception from the colorectum to the spinal cord of rodents. Electromyogram recordings were made from the transverse oblique abdominal muscles in anesthetized wild type (C57BL/6) mice and acute noxious intraluminal distension stimuli (100-120 mmHg) were applied to the terminal $15 \mathrm{~mm}$ of colorectum to activate visceromotor responses (VMRs). Lesioning the lumbar colonic nerves in vivo had no detectable effect on the VMRs evoked by colorectal distension. Also, lesions applied to the right or left hypogastric nerves failed to reduce VMRs. However, lesions applied to both left and right branches of the rectal nerves abolished VMRs, regardless of whether the lumbar colonic or hypogastric nerves were severed. Electrical stimulation applied to either the lumbar colonic or hypogastric nerves in vivo, failed to elicit aVMR. In contrast, electrical stimulation $(2-5 \mathrm{~Hz}, 0.4 \mathrm{~ms}$, $60 \mathrm{~V}$ ) applied to the rectum reliably elicited VMRs, which were abolished by selective lesioning of the rectal nerves. Dil retrograde labeling from the colorectum (injection sites 9-15 mm from the anus, measured in unstretched preparations) labeled sensory neurons primarily in dorsal root ganglia (DRG) of the lumbosacral region of the spinal cord (L6-S1). In contrast, injection of Dil into the mid to proximal colon (injection sites 30-75 $\mathrm{mm}$ from the anus, measured in unstretched preparations) labeled sensory neurons in DRG primarily of the lower thoracic level (T6-L2) of the spinal cord. The visceral pain pathway activated by acute noxious distension of the terminal $15 \mathrm{~mm}$ of mouse colorectum is transmitted predominantly, if not solely, through rectal/pelvic afferent nerve fibers to the spinal cord. The sensory neurons of this spinal afferent pathway lie primarily in the lumbosacral region of the spinal cord, between L6 and S1.

Keywords: sensory neuron, pain, afferent, nociceptor

\section{INTRODUCTION}

Patients with irritable bowel syndrome (IBS) have reduced threshold of pain and discomfort in the colon and rectum (Mertz et al., 1995). There is now abundant evidence that extrinsic spinal afferent nerves must transmit these nociceptive signals arising from the gastrointestinal (GI) tract to the spinal cord. However, there are a number of extrinsic nerve pathways that could potentially transmit nociceptive signals from the colorectum to the spinal cord and the contribution of these pathway(s) to transmission of nociceptive stimuli is currently unknown. If we wish to make progress in developing therapies that target colorectal pain, it is essential that we identify which sensory nerves detect noxious stimuli arising from the colorectum and the precise pathway that these afferents take in reaching the spinal cord.

Rodents are the most commonly used models to study mechanisms underlying visceral pain in the GI tract, because they reliably generate a stereotypical pain reflex in response to acute noxious colorectal distension, known as the visceromotor responses (VMRs; Larsson et al., 2003; Arvidsson et al., 2006). It is now clear that spinal afferents, whose cell bodies lie in the dorsal root ganglia (DRG) underlie the sensory pathway that conducts visceral pain signals from the rectum to the spinal cord (Grundy, 2002, 2004; Brierley et al., 2004; Furness, 2006; Spencer et al., 2008a; Song et al., 2009). However, due to difficulties in identifying extrinsic nerve tracks in small laboratory mammals such as mice, there have been no lesion studies to identify unequivocally which extrinsic afferent pathway(s) detect and transmit painful stimuli arising from the GI tract to the spinal cord.

In mammals, two distinct spinal afferent nerve pathways exist that can potentially transmit sensory information from the rectum and distal colon to the spinal cord. These are known as the lumbar colonic nerves (LCN)/lumbar splanchnic and sacral colorectal/pelvic nerves (Brierley et al., 2004). However, which of these pathways is important in the detection and transmission of visceral pain from the colorectum is unclear. In mouse, these two distinct sensory nerve pathways have been recently shown to possess at least five different classes of afferent fibers, each of which responds selectively to different stimuli (Brierley et al., 2004). In general, it is now known that the colorectal/pelvic pathway is a predominantly low threshold sensory pathway (Brierley et al., 2004; Spencer et al., 2008b; Feng et al., 2010), responding to low intensity mechanical stimulation, whereas the lumbar splanchnic 
pathway is predominantly a high threshold pathway, carrying only a small proportion of stretch sensitive (10\%) afferent fibers (Brierley et al., 2004).

In this study, we developed a novel in vivo preparation in which it was possible, for the first time, to identify and lesion (or stimulate) the major extrinsic nerve pathways between the colorectum and spinal cord, whilst eliciting VMRs to noxious colorectal distension. This allowed us to identify which extrinsic afferent nerve pathway(s) are required for the generation of VMRs elicited following noxious colorectal distension. Secondly, we have injected the retrograde neuronal tracer, DiI into a similar region of colorectum as distension stimuli were applied, to identify the location of the sensory neurons that underlie the VMRs from this region. We show that rectal/pelvic afferents are responsible for transmission of nociceptive information to the spinal cord following acute noxious distension of the colorectum. The cell bodies of the DRG sensory neurons in this pathway lie primarily in the lumbosacral region of the spinal cord.

\section{MATERIALS AND METHODS PROTOCOL FOR ACTIVATING VISCEROMOTOR RESPONSES}

C57BL/ 6 wild type mice 3-6 months of age were anesthetized by injection of pentobarbital sodium s.c. $(200-300 \mu \mathrm{L}$ of $6 \mathrm{mg} / \mathrm{mL})$. The depth of anesthesia was assessed by lack of response to hind limb or tail pinch. A collapsible balloon was inserted 4-7 $\mathrm{mm}$ from the anus and pressure was continuously monitored by a Gould pressure transducer, connected to a Powerlab recording system using Chart software (version 5.3, AD instruments, Sydney, Australia). When fully inflated, the balloon measured $8 \mathrm{~mm}$ in length and $7 \mathrm{~mm}$ in width. Thus, the maximum length of bowel distended by the balloon could be the terminal $15 \mathrm{~mm}$ of unstretched colorectum. Electromyographic (EMG) electrodes were placed into the left external oblique muscle and a reference electrode was placed in the quadriceps muscle of the opposing leg. EMG recordings were acquired at $20 \mathrm{kHz}$ and recorded on a PC running LabChart 6 Pro software, high pass filtered $(100 \mathrm{~Hz})$, and analyzed using Spike Histogram software (AD Instruments, Sydney, Australia). Intraluminal pressure was increased by raising a column of water from 0 to $100-120 \mathrm{mmHg}$ and maintained for $\sim 15-20 \mathrm{~s}$. In recent studies (Zagorodnyuk et al., 2011), we have found VMRs can be evoked in a near-linear fashion with increasing distension stimuli up to $120 \mathrm{mmHg}$. Therefore, to be sure nerve lesions had prevented all sensory signaling (both low and high threshold afferents) from different nerve pathways, we applied stimuli up to $100-120 \mathrm{mmHg}$. We were unable to detect any anatomical damage to the colorectum following stimulation up to $120 \mathrm{mmHg}$. Stimuli above $200 \mathrm{mmHg}$ were required to induce damage.

\section{TECHNIQUE TO LESION EXTRINSIC SPINAL NERVE PATHWAYS}

For experiments in which lesions were made to each of the major spinal nerve pathways, it was important to be able to visualize these nerves in vivo. Once mice had been fully anesthetized, a midline laparotomy was made to expose the internal organs. The small intestine and cecum were gently removed from the abdominal cavity, whilst retaining continuity with the vascular and nervous supplies. A fine cotton thread was looped around the distal colon at the junction of the inferior mesenteric artery
(IMA) and colon. This thread was then raised vertically, such that the distal colon was separated from the abdominal aorta by a space of approximately $3-4 \mathrm{~mm}$. This allowed sufficient space to place fine transmural stimulating wires across the IMA and all the LCN. It also allowed sufficient space to allow the LCN to be severed with ultra fine surgical dissecting scissors. To access the hypogastric nerves, the junction between the abdominal aorta and left and right iliac arteries was identified (see Figure 1; Miller and Szurszewski, 1994). The hypogastric nerves lie directly above (dorsal) to the left and right iliac arteries. This allowed clear visualization of the hypogastric nerves to facilitate lesions of the nerves, or allow direct access to stimulation by fine transmural electrical stimulation. To directly stimulate the hypogastric nerves using electrical stimulation, a fine $8 / 0$ suture thread was looped underneath each branch of the hypogastric nerves so that the nerve trunk was geographically separated from the iliac artery. This allowed transmural electrical stimulating wires to be placed around the nerve and not apply electrical stimuli to the iliac arteries themselves. To lesion the rectal nerves, a small incision was made through the skin caudal to the pelvic bone. The rectum was exposed and fine rectal nerves visualized. Once visualized, fine surgical scissors were used to lesion all rectal nerves between the anus and IMA.

\section{DiL RETROGRADE TRACING}

Mice of either sex were anesthetized with pentobarbital sodium (200-300 $\mu \mathrm{L}$ of $6 \mathrm{mg} / \mathrm{mL}$ ) and the depth of anesthesia was monitored by lack of response to a hind limb or tail pinch. Upon failure to elicit a tail or hind limb pinch, a midline laparotomy was made to expose the abdominal cavity. The ileum and cecum was gently reflected to one side of the abdominal cavity to expose the colon. The retrograde neuronal tracer, 1, 1' -didodecyl-3,3,3,3'tetramethylindocarbocyanine perchlorate (DiI C12 version), was injected into two sites along the colon: one site was a distance of $9-15 \mathrm{~mm}$ from the anus (when measured in unstretched preparations). This equated to a distance of $19-30 \mathrm{~mm}$ from the anus when the same segments of colorectum were removed from mice, stretched, and pinned taught in a petri dish. The second DiI injection site was made into the mid-proximal colon, a distance of 30-75 $\mathrm{mm}$ from the anus (measured in unstretched preparations), which equated to a distance of $60-100 \mathrm{~mm}$ from the anus when the same preparations were stretched and pinned taught. For DiI injections, a sterile 25 gauge needle connected to a Hamilton syringe ( $5 \mu \mathrm{L}$ maximum volume; Hamilton Company, Reno, NV, USA) was used to penetrate through the serosal surface. A series of two to four injection sites were made into the colonic musculature, and a total DiI injection volume of $2-5 \mu \mathrm{L}$ was made. A fine layer of gauze was used to wrap underneath the colon to prevent any leakage of DiI. The intestine and cecum was carefully repositioned back into the abdominal cavity and the abdominal muscles sutured closed. Mice were allowed to recover for a period of 7 days following dye injections, at which point mice were sacrificed. Following euthanasia, the colon and abdominal viscera were inspected for leakage of DiI. Any preparations showing potential spread or leakage of DiI were discarded from analysis. DRGs from bilateral spinal levels (T1-S3, inclusive) were then removed. DRGs were fixed in paraformaldehyde (4\%) 


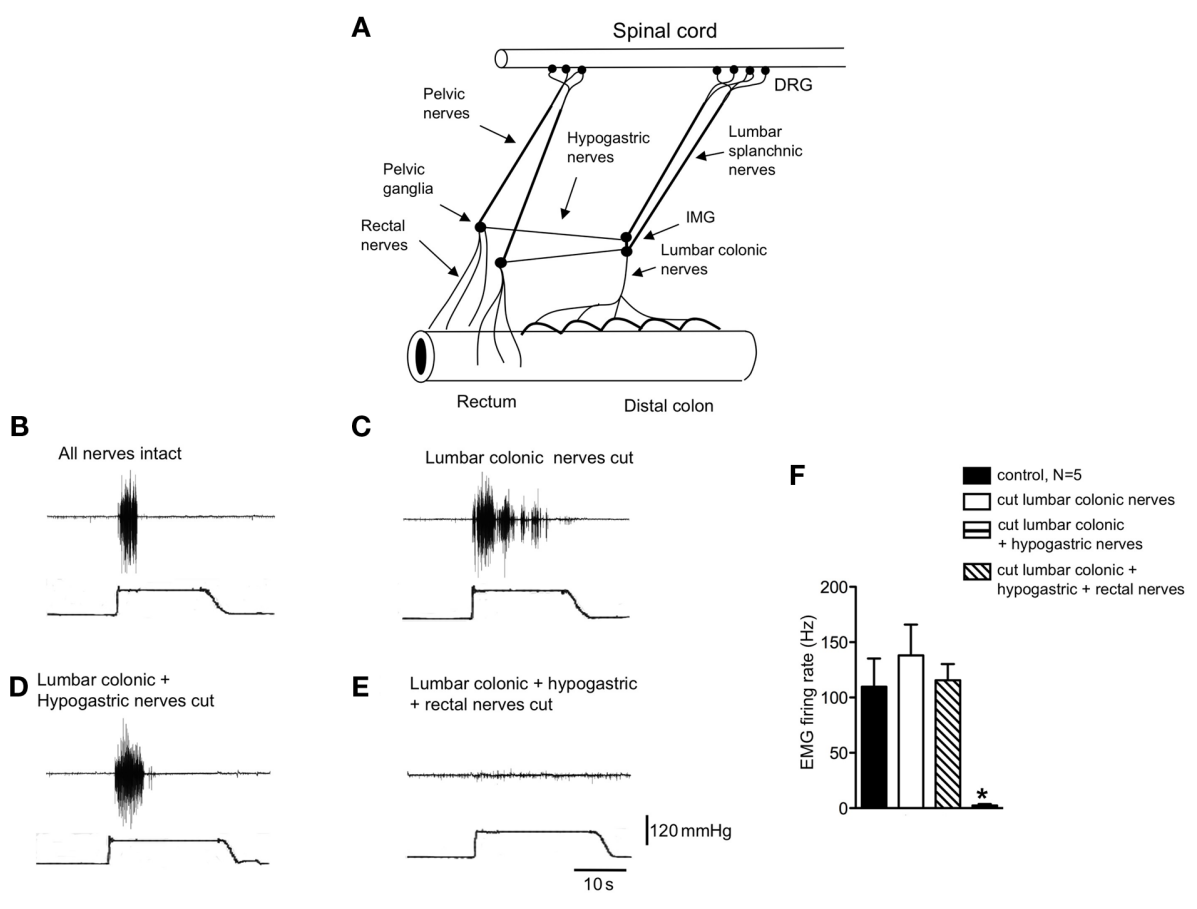

FIGURE 1 | Identification of the pain pathway underlying activation of the visceromotor responses to colorectal distension. (A) Diagrammatic representation of the major extrinsic nerve trunks that lie between the rectum and spinal cord. (B) Control visceromotor responses elicited by acute noxious colorectal distension. (C) Cutting only the lumbar colonic nerves did not reduced the VMRs to acute noxious colorectal distension. (D) Similar lesions applied to the hypogastric nerves also did not reduce the VMRs. (E) However, cutting of the rectal nerves abolished the visceromotor responses. (F) Shows the mean grouped data of VMRs evoked by acute noxious colorectal distension after lesions applied to the lumbar colonic, hypogastric, and rectal nerve trunks from $N=5$ animals. ${ }^{*}$ Refers to significant difference $(P<0.05)$. for 3 days before cryoprotection in sucrose for 1 day. DRG were cryostat-sectioned at $-19^{\circ} \mathrm{C}$ and $12 \mu \mathrm{m}$ sections made throughout all ganglia. Two sections (sections 5 and 9) from each DRG were retained and cover slip mounted. Fluorescent images of all DRG sections were photographed within 2 days to avoid spread and fading of DiI from filled nerve cell bodies. Because DRGs are autofluorescent, DiI-labeled cell bodies were discriminated by having fluorescence intensities three times above the background, which was defined by the mean fluorescence of neuronal cell bodies obtained from similar age mice that had not been injected with DiI. The mean number of labeled neurons was counted from the two sections obtained from each of the two DRGs obtained at each spinal segment (i.e., left and right DRG). All experimentation described above was approved by the Animal Welfare Committee at Flinders University of South Australia (approval no. 640/07).

\section{ANALYSIS AND STATISTICS \\ Data analysis}

Electromyogram recordings of the mean firing rate of action potentials was calculated during 10-s colorectal distensions. Results are expressed as means \pm SEM, with $N$ referring to the number of animals on which observations were made. Statistical analysis was performed by ANOVA (one-way) using Prism v.5 software (GraphPad Software, Inc., San Diego, CA, USA). Differences were considered significant if $P<0.05$.

\section{RESULTS IDENTIFICATION OF THE PAIN PATHWAY UNDERLYING THE VMR TO COLORECTAL DISTENSION}

In anesthetized mice, the abdominal cavity was exposed via midline laparotomy to allow access to the lumbar colonic, hypogastric nerves, and rectal nerves (Figure 1A). Nerve lesions were then applied systematically to each of the major spinal afferent pathways lying between the colorectum and spinal cord, whilst applying acute noxious colorectal distension stimuli (100-120 $\mathrm{mmHg}$ ) to assess their contribution to the transmission of visceral pain arising from the colorectum. Once reproducible VMRs were elicited (Figure 1B), a lesion was applied through the LCN. Lesions of the LCN had no detectable effect on the VMR to colorectal distension (Figures $\mathbf{1 C}, \mathbf{F}, N=9$ ). In these same animals in which the LCN had been severed, further sectioning of the left and right hypogastric nerves also had no effect on the VMRs to the same intensity stimulation $(N=5$; Figures 1D,F). However, further lesions applied to both left and right branches of the rectal nerves always abolished VMRs, even with maximal intensity distension (120 mmHg; Figures 1E,F, $N=9$ ). If lesions were applied to he rectal nerves, whilst preserving the LCN and hypogastric nerves, no VMRs could be elicited to colorectal distension $(N=3)$. This indicates that the major sensory pathway activated by noxious distension of this particular region of colorectum runs via the rectal and pelvic nerves to the spinal cord. 


\section{ELECTRICAL STIMULATION OF THE EXTRINSIC NERVES INNERVATING THE COLORECTUM}

We investigated whether transmural electrical stimulation applied to the extrinsic nerves between the colorectum and spinal cord would be able to elicit VMRs from each of the major nerve pathways. When fine transmural electrical stimulating wires were placed around the lumbar colonic or hypogastric nerves, no VMRs were evoked ( $5 \mathrm{~Hz}, 0.4 \mathrm{~ms}, 60 \mathrm{~V}, 5 \mathrm{~s}, N=6$ ). However, when the same stimulating electrodes were positioned around the exposed colorectum ( $5 \mathrm{~mm}$ from the anus), VMRs were reliably elicited (Figure 2A). Lesions applied to the rectal nerves abolished VMRs evoked by nerve stimulation ( $N=5$; Figure 2B). To confirm in these preparations that rectal nerve lesions were specific for the rectal nerves only, and had not prevented transmission along the spinal cord, we applied a tail pinch and hind limb, after the rectal nerves had been severed. In these cases, it was always possible to reliably evoke VMRs following tail pinch or hind limb pinch, when the rectal nerves had been severed and responses to colorectal stimulation abolished $(N=4$; Figure 2$)$.

\section{IDENTIFICATION OF THE EXTRINSIC SENSORY INNERVATION OF THE DISTAL COLON}

We paid particular attention to the site at which DiI was injected into the GI tract, measured as the distance from the anus in both unstretched and stretched segments of isolated colorectum. It was obvious that the distance at which DiI was injected from the anus changed considerably depending on whether the preparation was unstretched, or stretched and pinned taught in a dissecting dish.

We injected the retrograde neuronal tracer, DiI, into a similar region of colorectum that was distended by the intraluminal balloon. Following laparotomy, DiI was injected through the serosa and into the muscularis externa a distance of 9-15 mm from the anus (measured in unstretched preparations). These same injection sites measured a distance of $19-30 \mathrm{~mm}$ in stretched preparations. In control mice, it was found that the greatest number of DRG neurons were located in the sacral spinal cord at the level of L6 and S1, with a small proportion of neurons labeled in T13-L1 (see Figure 3). On average, a mean number of $17 \pm 6$ DRG neurons were labeled in L6 per section (Figure 3, $N=5$ ).

\section{IDENTIFICATION OF THE EXTRINSIC SENSORY INNERVATION OF THE MID TO PROXIMAL COLON}

We also injected DiI further from the anus, in this case in the region of the mid to proximal colon in a separate cohort. Injection sites were 30-75 $\mathrm{mm}$ from the anus in unstretched preparations (corresponding to a distance $60-100 \mathrm{~mm}$ in these same preparations stretched in vitro). The most striking difference was the relatively low number of retrogradely labeled neurons from the mid to proximal colon, despite the same volume of DiI injections and similar number of injection sites into the muscularis externa. The peak labeling of DRG was found to lie between T12 and L1 $(N=5)$, where the mean number of positively labeled neurons was $1.8 \pm 0.7$ at $\mathrm{T} 13(N=5)$ and $0.8 \pm 0.3$ neurons positively labeled at T9 (Figure $4, N=5$ ).

\section{DISCUSSION}

It is well known that the colorectum of mammals receives a complex extrinsic sensory innervation, via the lumbar splanchnic/lumbar colonic and rectal/pelvic afferent pathways. Even within each of these pathways, there are at least four or five different classes of afferent fiber (Brierley et al., 2004), that respond to different types of stimuli and different intensities of stimulation. Understanding which nerve pathway(s) transmit visceral pain from the colorectum to the spinal cord is of supreme importance, if we wish to

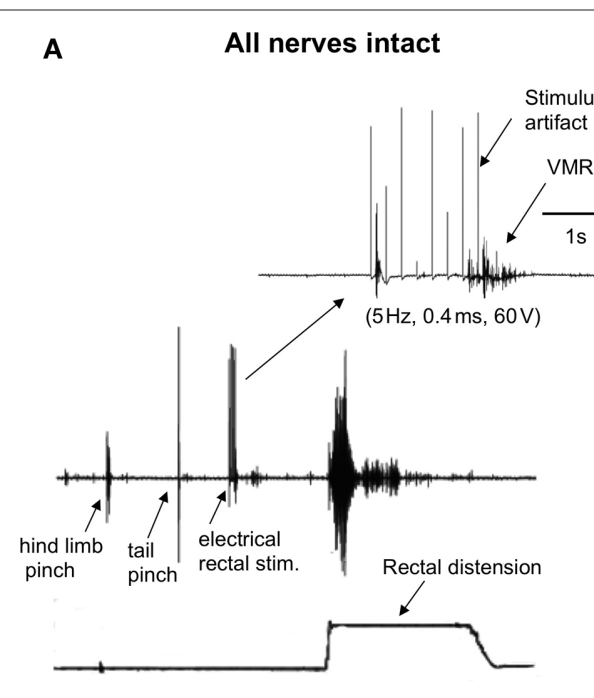

FIGURE 2 | Lesions to the rectal nerves selectively ablated responses to colorectal distension and electrical stimulation of the colorectum. (A) In an anesthetized mouse, a single hind limb pinch, tail pinch, electrical stimulation ( $5 \mathrm{~Hz}, 0.4 \mathrm{~ms}, 60 \mathrm{~V}, 5 \mathrm{~s}$ ) of the colorectum or acute noxious colorectal distension
B Rectal nerves only cut
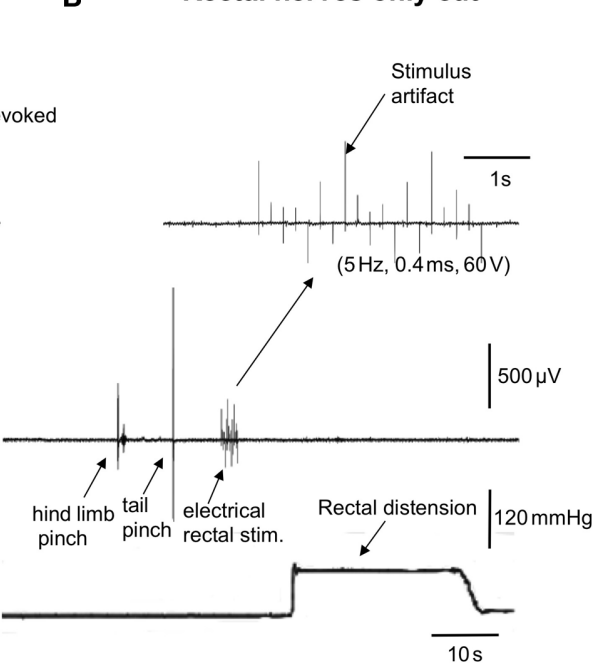

all elicited VMRs. (B) Lesions applied only to the left and right branches to the rectal nerves abolished VMRs to acute noxious colorectal distension and electrical stimulation of the rectum, but not those evoked by hind limb pinch or tail pinch. 


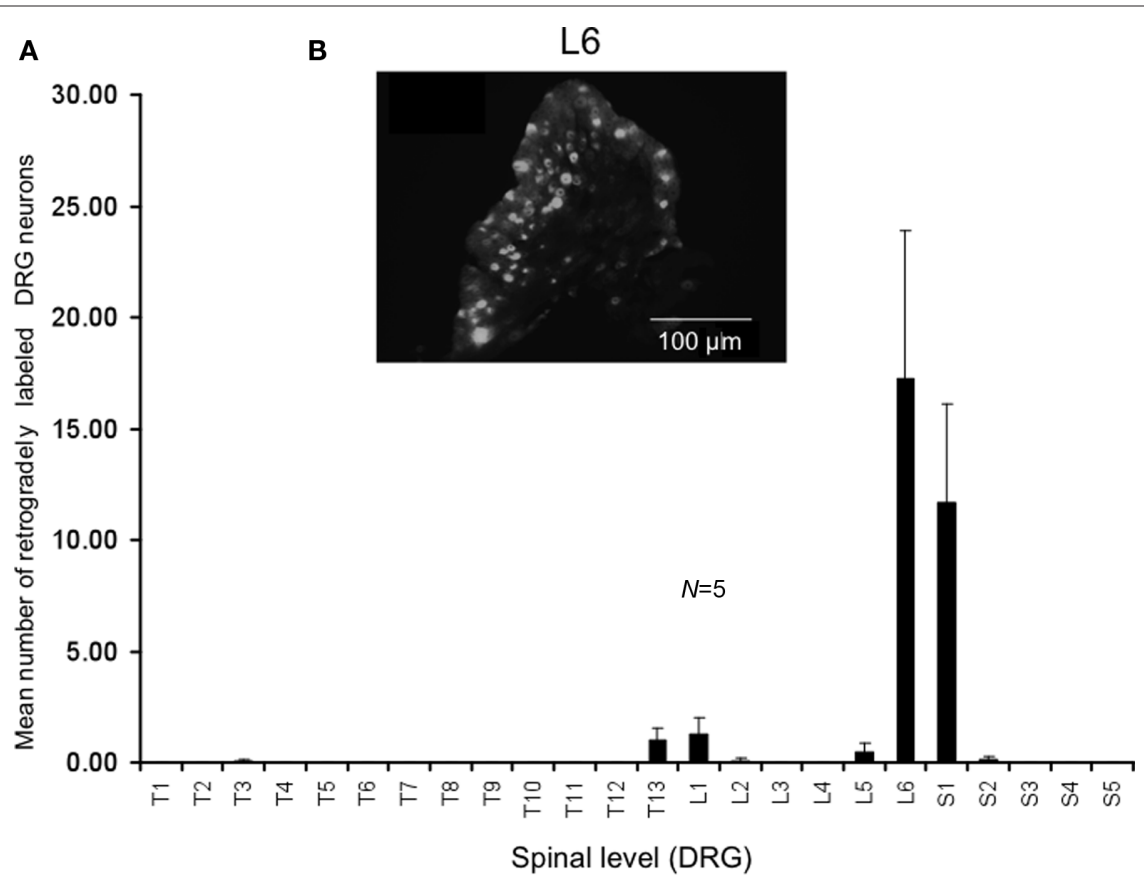

FIGURE 3 | Distribution of retrogradely labeled DRG neurons following injection of Dil into the mouse distal colon. (A) Following Dil injections made 9-15 $\mathrm{mm}$ from the anal sphincter (measured from unstretched colorectum preparations), the distribution of retrogradely labeled DRG neurons were identified. The majority of labeled neurons were identified in the lumbosacral region of spinal cord. Primary labeling was identified at levels of L6 and S1. (B) Fluorescence micrograph showing labeled neurons at L6. understand the origin and types of afferents important for signaling nociceptive stimuli to the central nervous system. In this study, we developed a novel preparation in anesthetized mice in which it was possible to visualize the major spinal afferent pathways that communicate between the colorectum and spinal cord, whilst applying noxious distension stimuli to the colorectum. This allowed us to systematically lesion each of the major afferent pathways to unequivocally determine which pathway(s) were required for signaling noxious distension stimuli from the colorectum.

Using this novel preparation, the major finding of the current study has shown the rectal/pelvic nerve pathway is essential for the detection and transmission of VMRs elicited by acute noxious distension of the terminal $15 \mathrm{~mm}$ of colorectum. This conclusion was based on the finding that lesions applied to the rectal nerves always abolished VMRs to colorectal distension, but lesions to the lumbar colonic or hypogastric nerves had no measurable effect.

\section{SENSORY PATHWAY INVOLVED IN DETECTION OF NOXIOUS COLORECTAL DISTENSION}

Identification of the nociceptive pathways from the terminal region of the GI tract has been a subject of much speculation (Brierley et al., 2009; Hughes et al., 2009; Feng et al., 2010). In our study, when DiI was injected into the distal colon, at a distance of 9-15 mm from the anus (measured in unstretched preparations), the sensory neurons retrogradely labeled were found at the level of the lumbosacral spinal cord (peak at L6-S1). This suggests that distension of the distal colon, at the transition zone between the rectal nerves and LCN utilizes sensory neurons primarily in the lumbosacral spinal cord, with only a small proportion from the thoracic spinal cord. This observation is highly consistent with the results from our lesion experiments which showed that lesions to the lumbar splanchnic pathways did not reduce VMRs following acute noxious colorectal distension, but were abolished by rectal nerve lesions.

A previous study by Robinson et al. (2004) injected fast blue into the descending colon of mice and also identified two peaks of DRG distribution, one peak at the level of T7 and the other at L3. It was stated that fast blue was injected into the descending colon via laparotomy, but it is not exactly clear how far in millimeters the fast blue was injected into the colon from the anus. One of the major findings of the study by Robinson et al. (2004) was that spinal afferents innervating the descending colon were largely located in thoracolumbar segments (T11-L1) segments. This appears to contrast with our findings, since we found predominantly thoracic labeling of DRG neurons when DiI was injected into the mid to proximal region of colon, at a distance of $30-75 \mathrm{~mm}$ from the anus (measured in unstretched preparations). We used the same strain and similar age of mouse as in the study of Robinson et al. (2004), but different neuronal tracers were used. It is possible, but seems difficult to reconcile how different tracers would reveal different distributions of DRG labeling.

In a study by Tan et al. (2008) cholera toxin B was injected into two sites in the mouse descending colon, $\sim 20 \mathrm{~mm}$ from the anus. In that study, it was reported that DRGs innervating this region were primarily located in T13-L1 and L4-S1. The results of Tan et al. (2008) are similar to our findings. Also, a study by Christianson et al. (2007) injected cholera toxin B into the mouse descending colon, at distances that were 10 and $20 \mathrm{~mm}$ from the anus. These 


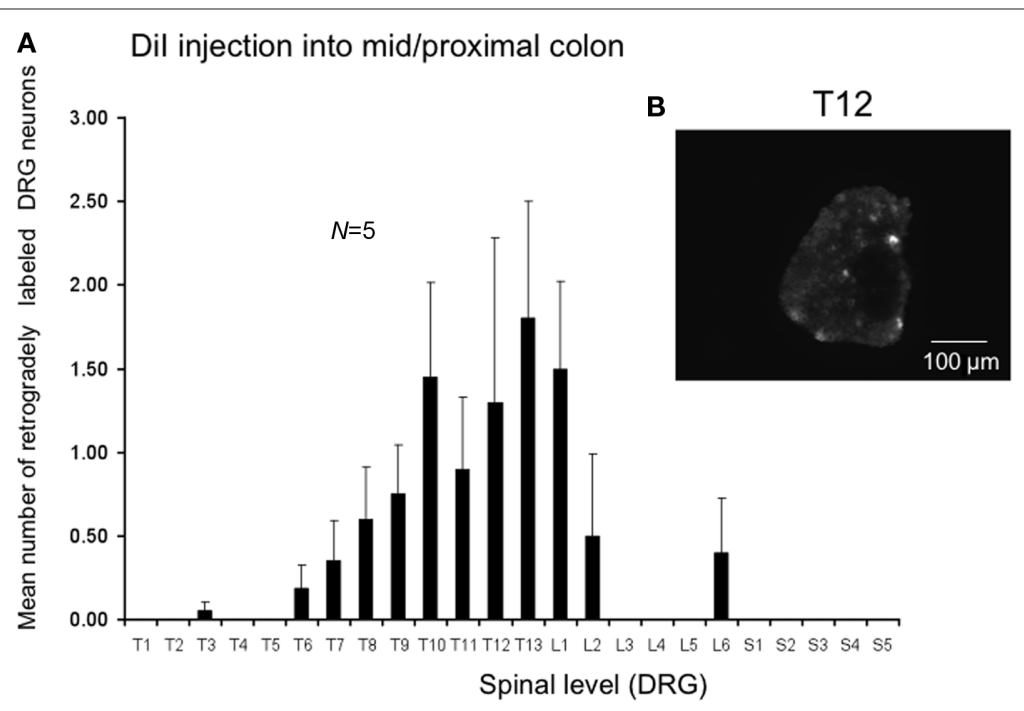

FIGURE 4 | Distribution of retrogradely labeled DRG neurons following injection of Dil into the mid to proximal colon. (A) Dil injected into the mouse colon 30-75 mm from the anus (measured in unstretched colon preparations, $N=5$ ). Dil labeled neurons were primarily identified between T5 and $\mathrm{L} 2$, with peak labeling between T11-L1. Only one to four neurons on average were labeled in any DRG section. (B) Shows a fluorescence micrograph of labeled neurons at T12.

investigators also found positively labeled DRG neurons were primarily located in the lumbosacral region, with only a small proportion of neurons labeled in thoracolumbar DRGs.

During our study, we also had an opportunity to assess functionally the relative contributions of thoracolumbar and sacral sensory pathways by severing acutely the major nerve trunks, selectively. As mentioned above, acute lesions to either, or both of the hypogastric nerves or the LCN had no detectable effect on the amplitude of VMRs. However, cutting the rectal nerves consistently abolished the response regardless of whether lumbar colonic or hypogastric nerves were severed. This strongly supports our retrograde tracing studies that show sacral afferents, traveling via rectal and pelvic nerves to the distal bowel, are the primary pathway involved in detecting acute noxious distension of the terminal rectum $(\sim 15 \mathrm{~mm}$ of unstretched rectum). This conclusion is further substantiated by the observation that VMRs could be reliably elicited following electrical stimulation of the rectal nerves, but not by the lumbar colonic or hypogastric nerves. Our conclusions that the VMRs elicited by acute noxious colorectal distension is transmitted via the rectal/pelvic afferents is remarkably similar to the findings reported by Nagabukuro and Berkley (2007), who distended the vagina to elicit VMRs. They also reported that lesions applied to the hypogastric nerves did not reduced VMRs to vaginal distension, but were abolished by pelvic nerve lesions.

\section{DENSITY OF DRG NEURONS INNERVATING COLORECTUM IS HIGHER THAN THAT THOSE OF MID-PROXIMAL COLON}

One of the major findings of this study was the distinct differences in density of DRG neurons labeled from DiI injections made in the colorectum compared with those made into

\section{REFERENCES}

Arvidsson, S., Larsson, M., Larsson, H., Lindstrom, E., and Martinez, V. (2006). Assessment of visceral pain-related

mid-proximal colon. Following colorectal injections of DiI the number of labeled neurons was approximately 20 times higher per DRG section, compared with DiI injections into the midproximal colon, whilst maintaining all other parameters constant (volume of dye injected, number of sites injected, time allowed for uptake, etc). On average, there were typically only about one to two labeled neurons per section from mid-proximal colon injections. The reason why the sensory innervation of the colorectum is considerably greater than the mid-proximal colon is not clear, but may be related to additional afferent nerve pathways recruited during the defecation reflex. The greater density of spinal afferents innervating the rectum conceptually seems similar to the increased density of intestinofugal neurons which also increases closer to the rectum (Messenger and Furness, 1992).

\section{CONCLUSION}

The results of this study show that the visceral pain pathway activated by acute noxious distension of the terminal $15 \mathrm{~mm}$ of colorectum is mediated via the rectal/pelvic spinal afferents, whose cell bodies lie primarily in the lumbosacral region of the spinal cord. No evidence was found to suggest that lumbar splanchnic afferents are required for the detection and transmission of noxious mechanical stimuli from this region of colorectum.

\section{ACKNOWLEDGMENTS}

We wish to acknowledge the financial support provided by the National Health and Medical Research Council (NH\&MRC) of Australia to grant numbers 535033 and 535034.

Brierley, S. M., Hughes, P. A., Page, A. J., Kwan, K.Y., Martin, C. M., O’Donnell, T. A., Cooper, N. J., Harrington, A. M. Adam, B., Liebregts, T., Holtmann,
G., Corey, D. P., Rychkov, G. Y., and Blackshaw, L.A. (2009). The ion channel TRPA1 is required for normal mechanosensation and is modulated 
by algesic stimuli. Gastroenterology 137, 2084-2095, e2083.

Brierley, S. M., Jones, R. C. III, Gebhart, G. F., and Blackshaw, L. A. (2004). Splanchnic and pelvic mechanosensory afferents signal different qualities of colonic stimuli in mice. Gastroenterology 127, 166-178.

Christianson, J. A., Liang, R., Ustinova, E. E., Davis, B. M., Fraser, M. O., and Pezzone, M. A. (2007). Convergence of bladder and colon sensory innervation occurs at the primary afferent level. Pain 128, 235-243.

Feng, B., Brumovsky, P. R., and Gebhart, G. F. (2010). Differential roles of stretch-sensitive pelvic nerve afferents innervating mouse distal colon and rectum. Am. J. Physiol. Gastrointest. Liver Physiol. 298, G402-G409.

Furness, J. B. (2006). The Enteric Nervous System. Oxford: Blackwell Publishing.

Grundy, D. (2002). Neuroanatomy of visceral nociception: vagal and splanchnic afferent. Gut 51(Suppl. 1), i2-i5.

Grundy, D. (2004). What activates visceral afferents? Gut 53(Suppl. 2), ii5-ii8.

Hughes, P. A., Brierley, S. M., and Blackshaw, L. A. (2009). Postinflammatory modification of colonic afferent mechanosensitivity. Clin. Exp. Pharmacol. Physiol. 36, 1034-1040.
Larsson, M., Arvidsson, S., Ekman, C., and Bayati, A. (2003). A model for chronic quantitative studies of colocolorectal sensitivity using balloon distension in conscious mice - effects of opioid receptor agonists. Neurogastroenterol. Motil. 15, 371-381.

Mertz,H., Naliboff, B., Munakata, J., Niazi, N., and Mayer, E. A. (1995). Altered colorectal perception is a biological marker of patients with irritable bowel syndrome. Gastroenterology 109, 40-52.

Messenger, J. P., and Furness, J. B. (1992). Distribution of enteric nerve cells that project to the coeliac ganglion of the guinea-pig. Cell Tissue Res. 269, 119-132.

Miller, S. M., and Szurszewski, J.H. (1994). "Physiology of prevertebral ganglia," in Physiology of the Gastrointestinal Tract, 4th Edn, Chapter 19, ed. L. R. Johnson (New York: Raven Press), 795-877.

Nagabukuro, H., and Berkley, K. J. (2007). Influence of endometriosis on visceromotor and cardiovascular responses induced by vaginal distention in the rat. Pain 132(Suppl. 1), S96-S103.

Robinson, D. R., McNaughton, P. A., Evans, M. L., and Hicks, G. A. (2004). Characterization of the primary spinal afferent innervation of the mouse colon using retrograde label- ling. Neurogastroenterol. Motil. 16, 113-124.

Song, X., Chen, B. N., Zagorodnyuk, V. P., Lynn, P. A., Blackshaw, L. A., Grundy, D., Brunsden, A. M., Costa, M., and Brookes, S. J. (2009). Identification of medium/high-threshold extrinsic mechanosensitive afferent nerves to the gastrointestinal tract. Gastroenterology 137, 274-284.

Spencer, N. J., Kerrin, A., Singer, C. A. Hennig, G. W., Gerthoffer, W. T., and McDonnell, O. (2008a). Identification of capsaicin-sensitive colorectal mechanoreceptors activated by colorectal distension in mice. Neuroscience 153 518-534.

Spencer, N. J., Kerrin, A., Zagorodnyuk, V. P., Hennig, G. W., Muto, M., Brookes, S. J., and McDonnell, O. (2008b). Identification of functional intramuscular colorectal mechanoreceptors in aganglionic colorectal smooth muscle from piebald lethal mice. Am. J. Physiol. Gastrointest. Liver Physiol. 294, G855-G867.

Tan, L. L., Bornstein, J. C., and Anderson, C. R. (2008). Distinct chemical classes of medium-sized transient receptor potential channel vanilloid 1-immunoreactive dorsal root ganglion neurons innervate the adult mouse jejunum and colon. Neuroscience 156, 334-343.
Zagorodnyuk, V. P., Kyloh, M., Nicholas, S., Peiris, H., Brookes, S. J., Chen, B. N., and Spencer, N. J. (2011). Selective loss of visceral pain in the rectum of an endothelin-3 deficient mouse model of Hirschsprung's disease. J. Physiol. (in press).

Conflict of Interest Statement: The authors declare that the research was conducted in the absence of any commercial or financial relationships that could be construed as a potential conflict of interest.

Received: 26 November 2010; accepted: 27 January 2011; published online: 22 February 2011.

Citation:KylohM, Nicholas S, Zagorodnyuk VP, Brookes SJ and Spencer NJ (2011) Identification of the visceral pain pathway activated by noxious colorectal distension in mice. Front. Neurosci. 5:16. doi: 10.3389/ fnins.2011.00016

This article was submitted to Frontiers in Autonomic Neuroscience, a specialty of Frontiers in Neuroscience.

Copyright $\odot 2011$ Kyloh, Nicholas, Zagorodnyuk, Brookes and Spencer. This is an open-access article subject to a nonexclusive license between the authors and Frontiers Media SA, which permits use, distribution and reproduction in other forums, provided the original authors and source are credited and other Frontiers conditions are complied with. 